

\title{
Packets of resonant modes in the Fermi-Pasta-Ulam system
}

\author{
Tommaso Genta $^{\mathrm{a}}$, Antonio Giorgilli ${ }^{\mathrm{b}}$, Simone Paleari ${ }^{\mathrm{b}}$, Tiziano Penati,* \\ ${ }^{a}$ Università degli Studi di Milano, Corso di Laurea in Matematica, \\ Via C.Saldini 50, 20133 - Milano, Italy. \\ ${ }^{b}$ Università degli Studi di Milano, Dipartimento di Matematica, \\ Via C.Saldini 50, 20133 - Milano, Italy.
}

\begin{abstract}
We reconsider the phenomenon of localization of energy in low frequency modes in the FPU system, exploiting the resonances in the lower part of the spectrum. Using the resonant normal form of Birkhoff we construct some candidates of approximate first integrals which we put in correspondence to packets of low frequency modes. By numerical calculation we show that the packet associated to the best quasi-integral involves all modes up to a frequency $\omega^{*}(\varepsilon)$, where $\varepsilon$ is the specific energy. The phenomenon disappears when the specific energy is bigger than a threshold value. The dependence of the relevant quantities on the number $N$ of particles is also investigated. A final section is devoted to a first comparison with the Toda model.
\end{abstract}

Keywords: Nonlinear chain, FPU model, Energy localization, Resonances, Thermodynamic limit 2010 MSC: 70K45, 70K30, 70H08, 70H09, 70H11, 37J40

\section{Introduction and summary of the results}

The FPU paradox, originated by the celebrated report of Fermi, Pasta and Ulam [11], has been the subject of many studies in the past decades. As is well known, the aim of Fermi, Pasta and Ulam was to observe the rate thermalization in a non linear chain of identical particles with nearest neighbour non linear interaction and fixed ends. However, the unexpected result was that no thermalization was observed, at least with the initial condition and the observation times of the FPU experiment. On the contrary, the energy remained concentrated only on a few modes. Further investigation has shown that the sharing of energy, if it happens, takes a very long time that may become unobservable even with the most powerful computers. The problem is to explain this phenomenon, and to prove or disprove its persistence in the thermodynamic limit.

Let us recall the model. Following the original FPU report, we consider a discretization of a string as a chain of $N+2$ identical particles on a segment, with fixed ends. The Hamiltonian of the system is written as

$H(x, y)=\sum_{j=1}^{N} \frac{y_{j}^{2}+x_{j}^{2}}{2}+\frac{\alpha}{3} \sum_{j=0}^{N}\left(x_{j+1}-x_{j}\right)^{3}+\frac{\beta}{4} \sum_{j=0}^{N}\left(x_{j+1}-x_{j}\right)^{4}$,

with $x_{0}=x_{N+1}=0$, and where $(x, y) \in \mathbb{R}^{2 N+2}$ are the coordinates and the momenta, and $\alpha$ and $\beta$ are real parameters. It

${ }^{*}$ Corresponding author: $\mathrm{Ph}$. n. +39-02-50316131; Fax n. +39-0250316090.

Email addresses: tomgenta@gmail . com (Tommaso Genta), antonio.giorgilli@unimi.it (Antonio Giorgilli),

simone.paleari@unimi.it (Simone Paleari),

tiziano.penati@unimi.it (Tiziano Penati)

Preprint submitted to see published version is well known that normal modes may be introduced via the canonical transformation

$$
\begin{aligned}
& x_{j}=\sqrt{\frac{2}{N+1}} \sum_{k=1}^{N} q_{k} \sin \frac{j k \pi}{N+1} \\
& y_{j}=\sqrt{\frac{2}{N+1}} \sum_{k=1}^{N} p_{k} \sin \frac{j k \pi}{N+1}
\end{aligned}
$$

where $(q, p) \in \mathbb{R}^{2 N}$ are the coordinates and the momenta of the normal modes. The Hamiltonian then takes the form of a system of independent harmonic oscillators with a nonlinear coupling, namely

$$
H(q, p)=H_{0}(q, p)+H_{1}(q, p)+H_{2}(q, p),
$$

where the unperturbed Hamiltonian is

$$
H_{0}(q, p)=\frac{1}{2} \sum_{k=1}^{N}\left(p_{k}^{2}+\omega_{k}^{2} q_{k}^{2}\right)
$$

and $H_{1}(q, p), H_{2}(q, p)$ are homogeneous polynomials of degree 3 and 4, respectively. The frequencies $\omega$ are

$$
\omega_{k}=2 \sin \frac{k \pi}{2(N+1)}, \quad k=1, \ldots, N .
$$

The quantities of interest, actually the ones considered in most previous works on this subject, are the energies $E_{k}=$ $\left(p_{k}^{2}+\omega_{k}^{2} q_{k}^{2}\right) / 2$ of the normal modes. According to the prediction of Statistical Mechanics the time averages of such quantities should tend to equipartition of energy, i.e., they should satisfy

$$
\lim _{t \rightarrow+\infty} \frac{1}{t} \int_{0}^{t} E_{k}(\tau) d \tau=\varepsilon,
$$


where $\varepsilon=E / N$ is the specific energy of the system and $E$ is the total energy. The failure to observe the limit in the FPU experiment constitutes the FPU paradox.

The considerable work produced by many people over a time span of more than half a century has not yet produced a satisfactory explanation of the FPU paradox. Early works of Izrailev and Chirikov [23] and of Bocchieri, Scotti Bearzi and Loinger [8] may be considered as the starting points of two diverging interpretations, both having as a common point the existence of an energy threshold below which the FPU phenomenon shows up. On the one hand one claims that the threshold vanishes in the thermodynamic limit, namely when the number $N$ of particles becomes very large [23]; on the other hand it is conjectured that the threshold remains positive and far from zero no matter how large is $N$ [8]. This is just the beginning of a long story, not yet finished. An historical account may be found in the volume [13], which contains several papers on the subject, and in particular in the contributions of Lichtenberg et al. [24] and of Benettin et al. [2] to that volume. A wide set of references can be found there.

In this letter we concentrate our attention on metastability phenomena that occur in the dynamics of the FPU chain. In particular we consider the phenomenon of natural packets of low frequency modes that has been observed in the paper of Berchialla et al. [5]. The main idea of that paper was to make quantitative the observation of Fermi, Pasta and Ulam that if the energy is initially given only to the first mode (i.e., the lowest frequency one) then the high frequency modes do not take part in the energy sharing. The phenomenon observed was that during the initial phase of the evolution the energy spreads among the first modes until a "natural packet" is formed, extending up to modes of frequency $\omega<\omega^{*}(\varepsilon)$. The numerical indication gives $\omega^{*}(\varepsilon) \sim \varepsilon^{1 / 4}$. The modes belonging to the packet may share energy in a chaotic way, as is suggested by the calculations in [19]. After that the evolution looks frozen for a long time (the relaxation time), until a further spreading shows up letting the energy to flow towards all modes and possibly to reach equipartition. In a subsequent paper [6] the relaxation time is evaluated to grow as $\exp \left(\varepsilon^{-1 / 4}\right)$ for a pure $\beta$-model and initial data in the low part of the spectrum, but not containing the first mode. For different choices of initial data, and for the $\alpha$ model, in [4] the authors find a different behaviour, namely a power law (see also [3] for the role of the coherence of the phases in the initial data); in [4], the role of the distance to the Toda system as a function of $\beta$ is also explored.

Although this "metastability scenario" is extremely rich and complex, with some of its details depending on the different possible choices on the system, some fairly satisfactory theoretical explanations are available concerning its initial stages, i.e. the creation of these natural packets. Among those we quote the continuous approximation approach (see e.g. [1, 25]) where the dynamics of the $\alpha$ model is controlled by integrable PDEs, namely the KdV. Instead of integrable PDEs, it should be possible to use integrable system of ODEs, like the Toda model (see [28] in this direction). A different technique involved the use of Lyapunov orbits (see, e.g., [12, 27], where the Lyapounov orbits are named Q-Breathers); actually, at very low energy, when the natural packet is very narrow, its energy spectrum matches almost perfectly the one of the Lyapunov orbit. For larger values of energy it is reasonable to substitute periodic orbits with quasi periodic ones, i.e. to use low dimensional tori, as in $[9,10]$. A similar attempt can be found in [18] where exponential stability of a low dimensional manifold is proved.

What is essentially missing in almost all the previous results (the only exception being [18]), is the explanation for the long time persistence of these meta stable states. One of the few papers in such a direction for the FPU system is [21], where moderately long time stability is proved using a modulated Fourier expansion; in particular their approximation is valid over a longer time scale than [1] at the price of a narrower range of allowed energies.

Although the resonances are a key ingredient in some of these papers (like in [1] where a couple of $\mathrm{KdV}$ are obtained by means of a resonant normal form), we think they have not been completely exploited in order to understand long time behaviour.

In the present letter we investigate the phenomenon of natural packets by taking into account the resonances that characterize the first part of the FPU spectrum. For, the first part of the spectrum is close to a linear one, i.e., the frequencies are approximately $\omega_{2} \simeq 2 \omega_{1}, \omega_{3} \simeq 3 \omega_{1}, \ldots$. Thus it seems natural to imagine that a small change of the frequencies due to the non linearity may cause two or more modes to fall into a resonance, and this phenomenon is expected to be enhanced when energy is increased, so that more and more modes are involved. This corresponds to the phenomenology that is numerically observed for the natural packets.

The basic theoretical tool is the construction of a resonant Birkhoff normal form and of a corresponding formal first integral $\Phi$ in the form of a series expansion. We avoid here entering the detailed mathematical treatment of the problem since it requires quite boring calculations and involves subtle difficulties due to resonances with high frequency modes. We just recall that in many cases the theory allows us to identify quantities that remain almost invariant for very long times, e.g., $\varepsilon^{-r}$ for some high $r$ or even $\exp \left(\varepsilon^{-a}\right)$ with some positive $a<1$ (e.g., $1 / 4$ or $1 / 8$ or so).

We perform instead a numerical study based on the calculation of both the zeroth order approximation (actually a linear combination of the harmonic energies that is a polynomial of degree 2) that we call $\Phi_{0}$, and a first order nonlinear approximation, that we call $\Phi_{1}$ and which is hopefully better than $\Phi_{0}$. We analyze in particular the fluctuation of the two functions $\Phi_{0}$ and $\Phi_{1}$. Our conclusions are the following.

(i) For low specific energy and for a given number $N$ of particles we can identify an optimal value $n_{r}(\varepsilon)$, increasing with $\varepsilon$, which minimizes the fluctuation of the approximate first integral; we may consider this value as an indication of the width of the resonant packet.

(ii) Still for fixed $N$, there is a critical value $\varepsilon^{*}$ of the specific energy above which the behaviour of $\Phi_{1}$ becomes worse than that of $\Phi_{0}$ alone. In view of the general theory this may be considered as a threshold for the existence 
of metastability phenomena.

(iii) The results above appear to persist when the number $N$ of particles is increased. In particular, the length $n_{r}$ of the packet is proportional to the number $N$ of particles, which means that the packet extends up to a frequency $\omega_{r}(\varepsilon)$ independent of $N$.

Thus, our results suggest that the phenomenon of the natural packets may be interpreted as an effect of the resonance among the low frequency modes.

It might be interesting to extend our calculation to higher order terms in the expansion of $\Phi$, but the number of coefficients to be calculated grows so large with the number $N$ of degrees of freedom that the actual calculation is precluded.

The paper is organized as follows. In sect. 2 we give a short account of the theoretical background concerning the construction of Birkhoff's normal form in our case. In sect. 3 we present the results of our calculations.

\section{Resonant first integrals}

We recall here a few basic facts about the resonant Birkhoff's normal form. Complete expositions of the formal part of this theory may be found in Birkhoff's treatise [7] or in many papers like, e.g., [20] or [17]. The asymptotic properties of the normal form leading to exponential estimates of Nekhoroshev's type may be found in [16]. For an introductory exposition of the problem related to exponential stability see, e.g., [15].

\subsection{Resonant Birkhoff's normal form in a nutshell}

We consider a perturbed system of harmonic oscillators with Hamiltonian expanded in Taylor series as

$$
\begin{gathered}
H(x, y)=H_{0}(x, y)+H_{1}(x, y)+H_{2}(x, y)+\ldots, \\
H_{0}(x, y)=\frac{1}{2} \sum_{l=1}^{N} \omega_{j}\left(y_{j}^{2}+x_{j}^{2}\right),
\end{gathered}
$$

and with $H_{1}, \ldots$ homogeneous polynomials of degree $3,4, \ldots$ respectively. By the way, the Hamiltonian (3) may be given this form via the canonical transformation $x_{j}=\frac{x_{j}^{\prime}}{\sqrt{\omega}_{j}}, y_{j}=\sqrt{\omega}_{j} y_{j}$. In order to understand the meaning of the normal form it is convenient to use action-angle variables $I_{j}, \phi_{j}$ introduced by the canonical transformation

$$
x_{j}=\sqrt{2 I_{j}} \cos \phi_{j}, \quad y_{j}=\sqrt{2 I_{j}} \sin \phi_{j} .
$$

The unperturbed Hamiltonian in these variables takes the simple form $H_{0}=\sum_{l} \omega_{l} I_{l}$, while the nonlinear term $H_{s}$ becomes a trigonometric polynomial of degree $s+2$ in the angles $\phi$ with homogeneous polynomials of degree $s+2$ in $I_{1}^{1 / 2}, \ldots, I_{N}^{1 / 2}$ as coefficients.

Birkhoff's normal form is defined as follows. Let the resonance module $\mathcal{M}_{\omega}$ associated to the frequency vector $\omega$ be defined as

$$
\mathcal{M}_{\omega}=\left\{k \in \mathbb{Z}^{N}:\langle k, \omega\rangle=0\right\}
$$

where $\langle k, \omega\rangle=\sum_{l} k_{l} \omega_{l}$. It is well known (and easy to check) that $\mathcal{M}_{\omega}$ is a discrete subgroup of $\mathbb{Z}^{N}$. If $\mathcal{M}_{\omega}=\{0\}$, i.e., it is the trivial module, then the system is said to be a non resonant one, else it is said to be resonant with multiplicity $\operatorname{dim} \mathcal{M}_{\omega}$, the dimension of the resonance module. The Hamiltonian (7) is said to be in Birkhoff's normal form with respect to the resonance module $\mathcal{M}_{\omega}$ in case the functions $H_{1}(I, \phi), H_{2}(I, \phi), \ldots$ depend only on $I_{1}, \ldots, I_{N}$ and on integer combinations of the angles $\langle k, \phi\rangle$ with $k \in \mathcal{M}_{\omega}$. It is an easy matter to check that any function

$$
\Phi^{(\alpha)}=\sum_{l=1}^{N} \alpha_{l} I_{l}, \quad \alpha \perp \mathcal{M}_{\omega}
$$

is a first integral for a Hamiltonian in Birkhoff's normal form: just calculate the Poisson bracket of $\Phi^{(\alpha)}$ with $H$ and check that $\left\{H, \Phi^{(\alpha)}\right\}=0$. There are $N-\operatorname{dim} \mathcal{M}_{\omega}$ independent first integrals of the form above. In particular, if $\operatorname{dim} \mathcal{M}_{\omega}=0$ then the actions $I_{1}, \ldots, I_{N}$ are first integrals.

A classical result is that any Hamiltonian of the form (7) may be given a Birkhoff's normal form via a near the identity canonical transformation that can be written as a formal series expansion

$$
\begin{aligned}
& x_{l}=x_{l}^{\prime}+f_{l, 1}\left(x^{\prime}, y^{\prime}\right)+f_{l, 2}\left(x^{\prime}, y^{\prime}\right)+\ldots, \\
& y_{l}=y_{l}^{\prime}+g_{l, 1}\left(x^{\prime}, y^{\prime}\right)+g_{l, 2}\left(x^{\prime}, y^{\prime}\right)+\ldots,
\end{aligned}
$$

where the functions $f_{s}\left(x^{\prime}, y^{\prime}\right)$ and $g_{s}\left(x^{\prime}, y^{\prime}\right)$ are homogeneous polynomials of degree $s+1$. However, the transformation is only a formal one in the sense that the coefficients of the expansions can be determined with an algebraic procedure, but in general the series so obtained fail to be convergent. A quantitative analysis shows that in general the series have an asymptotic character. That is, in a ball of radius $\rho$ around the origin the size of the functions $f_{s}, g_{s}$ grows as $s !^{\tau} \rho^{s+1}$ with some $\tau>1$. Meaningful results can however be found by truncating the normalization procedure after a finite number $r \geq 1$ of steps, so that the Hamiltonian takes the form

$$
\begin{aligned}
H^{(r)}\left(x^{\prime}, y^{\prime}\right)=H_{0}\left(x^{\prime}, y^{\prime}\right) & +Z_{1}\left(x^{\prime}, y^{\prime}\right)+\ldots+ \\
& +Z_{r}\left(x^{\prime}, y^{\prime}\right)+\mathcal{R}^{(r)}\left(x^{\prime}, y^{\prime}\right),
\end{aligned}
$$

where the functions $Z_{1}, \ldots, Z_{r}$ are in normal form, while $\mathcal{R}^{(r)}$ is an unnormalized remainder in the form of a series starting with terms of degree $r+3$. According to the general theory this may be done with a generating function which is a polynomial of degree $r+2$. The form of the transformation (11) depends on the choice of $r$, and we keep trace of this fact by denoting the new variables as $x^{(r)}, y^{(r)}$, with $x^{(0)}=x, y^{(0)}=y$.

The Hamiltonian (12) possesses approximated first integrals that are found by writing $\Phi^{(\alpha)}$ as a function of the new variables $x^{(r)}, y^{(r)}$, i.e, $\Phi^{(\alpha)}=\sum_{l} \alpha_{l}\left(y_{l}^{(r)^{2}}+x_{l}^{(r)^{2}}\right) / 2$. For such a function one gets $\dot{\Phi}^{(\alpha)}=\left\{\Phi^{(\alpha)}, \mathcal{R}^{(r)}\right\}$ and a quantitative estimate gives

$$
\left|\dot{\Phi}^{(\alpha)}\right| \sim r !^{\tau} \rho^{r+3}
$$

The exponential estimate of Nekhoroshev's type is found by choosing an optimal truncation order $r_{\mathrm{opt}} \sim \rho^{-1 / \tau}$, which gives

$$
\left|\dot{\Phi}^{(\alpha)}\right| \sim \exp \left(\rho^{-1 / \tau}\right)
$$


Thus, a significant change of the approximate first integral may occur only after an exponentially long time.

In the FPU case the orbits are bounded in a neighbourhood of the origin, due to energy conservation. In rough terms we may consider a ball of radius $\rho \sim \sqrt{\varepsilon}$, although in some directions (e.g., all the energy in a single normal mode) the distance from the origin may approach $\sqrt{N \varepsilon}$.

On the basis of the theory sketched above we consider a minor extension of Birkhoff's theory by considering a resonance module $\mathcal{M}$ which takes into account the cases in which $|\langle k, \omega\rangle|$ is very small. The construction of the normal form remains consistent provided the chosen resonance module $\mathcal{M}$ includes $\mathcal{M}_{\omega}$, the resonance module generated by the frequency vector, i.e., if $\langle k, \omega\rangle \neq 0$ for all $k \in \mathbb{Z}^{N} \backslash \mathcal{M}$. The resulting transformed Hamiltonian is in normal form with respect to the module $\mathcal{M}$, and possesses $N-\operatorname{dim} \mathcal{M}$ formal first integrals of the form (10).

\subsection{The FPU case}

Let us now come to our use of the normal form for the FPU model. In order to take into account the quasi resonances of the lower part of the spectrum we pick an arbitrary number $n$ of resonant modes and consider the resonance module generated by the $n-1$ rows of the matrix

$$
\mathcal{M}_{n}=\left(\begin{array}{cccccccc}
-2 & 1 & 0 & 0 & 0 & 0 & \ldots & 0 \\
-3 & 0 & 1 & 0 & 0 & 0 & \ldots & 0 \\
\vdots & \vdots & \vdots & \ddots & \vdots & 0 & \vdots & \vdots \\
-n & 0 & 0 & 0 & 1 & 0 & \ldots & 0
\end{array}\right)
$$

corresponding to the quasi resonances $l \omega_{1}-\omega_{l} \sim 0$. Then we determine the canonical transformation

$$
x_{l}=x_{l}^{\prime}+f_{l, 1}\left(x^{\prime}, y^{\prime}\right)+\ldots, \quad y_{l}=y_{l}^{\prime}+g_{l, 1}\left(x^{\prime}, y^{\prime}\right)+\ldots
$$

which gives the truncated normal form

$$
H^{(1)}=H_{0}\left(x^{\prime}, y^{\prime}\right)+Z_{1}\left(x^{\prime}, y^{\prime}\right)+\mathcal{R}^{(2)}\left(x^{\prime}, y^{\prime}\right)
$$

(which corresponds to $r=1$ in (12)). Using computer algebra we calculate the coefficients of the polynomials $f_{j, 1}\left(x^{\prime}, y^{\prime}\right)$ and $g_{j, 1}\left(x^{\prime}, y^{\prime}\right)$, which have degree 2 , and forget terms of higher degree that are represented by dots in the formula above. Details concerning the actual implementation of an algorithm for constructing the normal form are given in [14]. The transformation (14) may be inverted as

$$
x_{l}^{\prime}=x_{l}-f_{l, 1}(x, y)+\ldots, \quad y_{l}^{\prime}=y_{l}-g_{l, 1}(x, y)+\ldots,
$$

still keeping only terms of degree 2 . An approximate first integral for the Hamiltonian (14) is

$$
\Phi_{1}^{(\alpha)}=\sum_{l=1}^{n} \alpha_{l}\left(y_{l}^{\prime 2}+x_{l}^{\prime 2}\right), \quad \alpha_{l}=l \quad \text { for } l=1, \ldots, n
$$

We shall also consider the zeroth order approximation of the first integral

$$
\Phi_{0}^{(\alpha)}=\sum_{l=1}^{n} \alpha_{l}\left(y_{l}^{2}+x_{l}^{2}\right), \quad \alpha_{l}=l \quad \text { for } l=1, \ldots, n .
$$

Since $\alpha$ is trivially determined by $n$, in the rest of the paper we either drop the superscript $(\alpha)$, or substitute it with $(n)$ to stress the number of resonances involved.

Suppose now for a moment that an exact first integral $\Phi^{\left(n_{r}\right)}$ exists for some number $n_{r}$ of resonances. That is, suppose the Birkhoff normal form with the resonance module $\mathcal{M}_{n_{r}}$ of dimension $n_{r}-1$ turns ot to be convergent. In that case our construction gives two successive approximation $\Phi_{0}$ and $\Phi_{1}$ of the exact first integral. If we let the system evolve and look $\Phi_{0}(t)$ and $\Phi_{1}(t)$ as functions of time then we expect to observe some fluctuation around an average value. The fluctuation is expected to be smaller for $\Phi_{1}$ than for $\Phi_{0}$ (and to become smaller and smaller if we could consider further approximations $\Phi_{2}, \ldots$ ). The problem is twofold: (i) such a first integral, if any, may exist only as a quantity conserved over exponentially long time, in Nekhoroshev sense; (ii) we have no indication a priori on the number $n_{r}$ of resonances to be considered. Concerning the point (i), according to Nekhoroshev's theory the expansion of the first integral should be truncated at an optimal order which grows very large when $\varepsilon$ is decreased. As a matter of fact we can determine only a low order approximation of such a function. Concerning the point (ii), $n_{r}$ is the quantity that we want to investigate numerically.

Exploiting the fact that the approximated first integrals $\Phi_{0}$ and $\Phi_{1}$ may be explicitly calculated we proceed as follows. For fixed $N$ and $\varepsilon$ we look for a value $n_{r}(N, \varepsilon)$ which minimizes the fluctuation of either $\Phi_{0}(t)$ or $\Phi_{1}(t)$. To this end we let the number $n$ of resonances to vary from 1 to $N-1$ and for every $n$ we calculate by numerical integration $\Phi_{0}^{(n)}(t)$ and $\Phi_{1}^{(n)}(t)$ together with the corresponding time average and variance, the superscript being introduced in order to take into account the number of resonances. If an optimal first integral $\Phi_{*}$ (in Nekhoroshev sense) exists for some $n_{r}$ then we must observe a fluctuation due to the difference $\Phi_{*}-\Phi_{j}^{(n)}$ between the optimal first integral and our approximation. In view of the trivial identity $\Phi_{*}=\Phi_{j}^{(n)}+\left(\Phi_{j}^{\left(n_{r}\right)}-\Phi_{j}^{(n)}\right)+\left(\Phi_{*}-\Phi_{j}^{\left(n_{r}\right)}\right)$ we may identify two different sources of fluctuation, namely: (i) the difference $\Phi_{j}^{\left(n_{r}\right)}-\Phi_{j}^{(n)}$, due to the bad choice of $n$, which is the dominant one for $n<n_{r}$ being due to the quadratic part of the function, but vanishes when $n=n_{r}$; (ii) the difference $\Phi_{*}-\Phi_{j}^{\left(n_{r}\right)}$ which is due to the truncation of the expansion of the optimal first integrals. We call the latter contribution the residual fluctuation. This is a fluctuation that can not be removed, but is expected to be smaller for $\Phi_{1}$ than for $\Phi_{0}$ (and would become smaller and smaller if higher order approximations could be constructed): in particular one can expect it to be of the order of $\varepsilon^{1 / 2}$ for $\Phi_{0}$, and of the order of $\varepsilon$ for $\Phi_{1}$. Thus, at fixed order $j$, the optimal number $n_{r}$ of resonances is the one which minimizes the fluctuation. Actually, if the initial conditions are chosen so that all the energy is concentrated within the first $n_{r}$ modes, and the dynamics maintains such a confinement, then the fluctuation may be expected to remain almost constant for $n>n_{r}$.

As to the dependence of $n_{r}$ on $\varepsilon$, we should expect that increasing the specific energy will increase the number of modes involved in the resonance. However, we can explore such a dependence only numerically, since presently we have no theo- 
retical indication.

The dependence of $n_{r}$ on the length $N$ of the chain is even more delicate, because the theory of Birkhoff's normal form usually gives quite bad predictions. However, previous works (quoted in the introduction) show that some metastability phenomena may persist in the thermodynamic limit, so that this aspect deserves to be investigated, too.

We close this section with the remark that higher order approximations could be considered in a straightforward way, but the calculation becomes unpractical for systems with, e.g., 127 or 255 particles. For, in order to construct the normal form up to degree $r$ we must calculate a generating function of degree $r+2$, which contains $\left(\begin{array}{c}2 N+r+2 \\ r+2\end{array}\right)$ coefficients. It is immediately seen that such a number grows very fast with $N$. E.g., in order to calculate $\Phi_{2}$ for $N=127$ one should compute a generating function with $1.7 \times 10^{8}$ coefficients, repeating the calculation for all the wanted values of the number $n$ of resonances. On the other hand, we are interested in increasing the value of $N$ in order to have some insight on the thermodynamic limit. Previous experience shows that some parameters may reach reasonably stabilized values only for quite large values of $N$ (see, e.g., [6]).

\section{Numerical results}

We perform all ${ }^{1}$ the calculations for the model (1) with $\alpha=$ $\beta=1 / 4$. The quartic term of the potential is added in order to avoid breaking of the chain when the number of particles, and so the total energy, is increased, but of course it has no effect on the calculation of the normal form and of the approximate first integrals up to degree 3 . The number of particles is chosen to be $15,31,63$, and 127 , in order to study the dependence on $N$. Concerning initial condition we considered two cases. In the first one we follow [11], i.e. all the energy is placed on the first normal mode; all the pictures shown refer to this situation. In the second case the initial conditions are chosen so that the first $N / 16$ modes are excited with energy linearly decreasing to zero. As mentioned in [5] this assures that the time needed in order to form the natural packet does not increase with $N$.

For $1 \leq n<N$ we construct the generating function that gives the Hamiltonian the normal form with respect to the resonance module $\mathcal{M}$ as in (13). Remark that for every $n$ the calculation must be performed from scratch.

By numerical integration we calculate the values at time $t$ of the coordinates of the normal form as given by (16), and so also the time evolution of the functions $\Phi_{0}(t)$ and $\Phi_{1}(t)$ as given by (18) and (17) (we omit the dependence on the choice of $n$, which however should be kept in mind). Then we determine the time average

$$
\langle\Phi\rangle_{T}=\frac{1}{T} \int_{0}^{T} \Phi(t) d t
$$

and the corresponding variance

$$
\sigma^{2}=\frac{1}{T} \int_{0}^{T}\left(\Phi(t)-\langle\Phi\rangle_{T}\right)^{2} d t
$$

\footnotetext{
${ }^{1}$ the only exception is represented by the last section where we used also a different value of $\beta$ for the comparison with the Toda model.
}

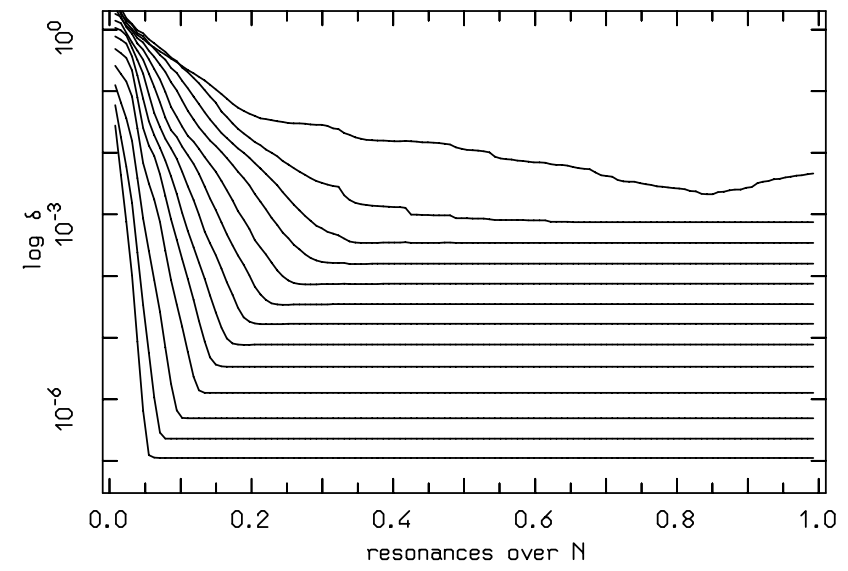

Figure 1: $\log \delta$ vs. $v=n /(N+1)$ for 13 values of the specific energy, logaritmically spaced from $\varepsilon=10^{-6}$ (bottom line) to $\varepsilon=10^{-2}$ (top line). Here $N=127$ and $T=10^{5}$. The function considered is $\Phi_{1}$.

We actually consider the relative fluctuation

$$
\delta=\frac{\sigma}{\langle\Phi\rangle_{T}} .
$$

Again, we omit the dependence on $n$ in the notation.

Let us come to the results, in the case of all the energy on the first normal mode. The dependence of the relative fluctuation $\delta$ on $v=n /(N+1)$ is illustrated in fig. 1 , where $n$ is the number of resonances taken into account. The data refer to 13 different values of the specific energy $\varepsilon$. It is seen that for small energies the fluctuation decreases almost exponentially up to some value $v_{r}(\varepsilon)$, and then remains practically constant. The value $n_{r}=$ $v_{r}(N+1)$ is the number of resonances that we are looking for. This nice behaviour tends to disappear as the specific energy is increased, as expected. Moreover, looking at the same curves at different times, one sees that after a transient the curves for low energies remain quite stable (for a long time), while for higher energies the right part of the curve steadily grows, and a unique minimum of the fluctuation is hardly identified.

The results in fig. 1 suggests that for a given (not too large) $\varepsilon$, a good fit of the plot may be given by a function

$$
\delta(v)= \begin{cases}e^{\gamma v} & \text { for } v \leq v_{r} \\ \eta & \text { for } v>v_{r}\end{cases}
$$

where $\gamma, \eta$ and $v_{r}$ are constants that can be determined numerically using the least squares method. The value of $v_{r}$ (and so of $n_{r}=v_{r}(N+1)$ ) so found obviously gives the wanted number of resonances for low energies, where the minimum of the fluctuation is easily identified. In the rest of the paper we will set $\Phi:=\Phi_{1}^{\left(n_{r}\right)}$, to denote our approximate resonant integral. We use the same criterion in order to produce a value for $v_{r}$ also for higher energies at a fixed time, whatever it means (see comments below).

We found particularly interesting the behaviour of the residual fluctuation $\eta$ as a function of the specific energy $\varepsilon$, whose plot is reported in fig. 2. Here the results for $\Phi_{0}$ and $\Phi_{1}$ are quite different at low energy, thus indicating that $\Phi_{1}$ is really a 


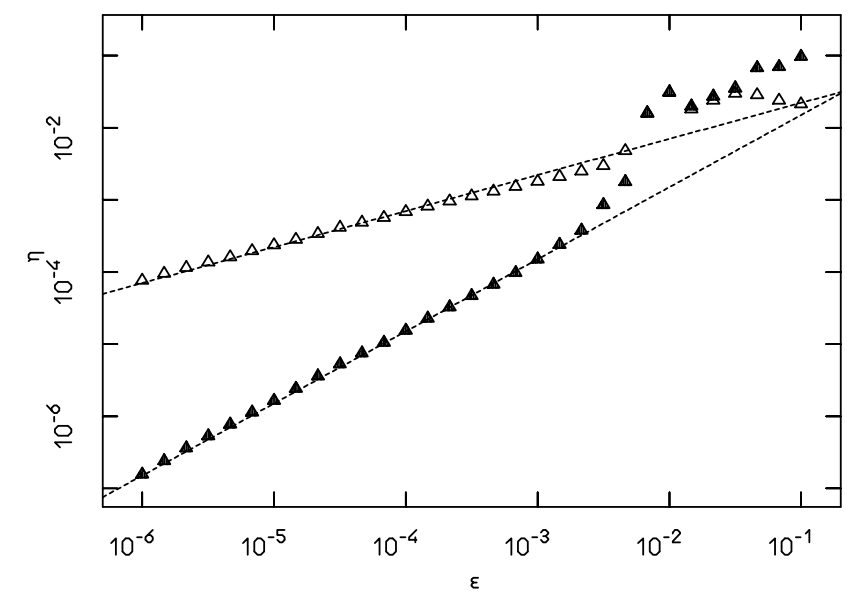

Figure 2: The residual fluctuation $\eta$ vs. $\varepsilon$. Here $N=127$ and $T=4 . \times 10^{5}$. The data refer to $\Phi_{0}$ (open triangles) and $\Phi_{1}$ (solid triangles). The dashed lines have slope respectively $1 / 2$ and 1 .

better approximation of resonant first integral. For higher energy the two curves tend to coalesce, which means that adding the cubic terms to the first integral does not actually improve its behaviour, and for even higher values the fluctuation of $\Phi_{1}$ overcomes that of $\Phi_{0}$, consistently with the theoretical prediction. This gives a rough numerical indication of the existence of a threshold, above which the packet of resonant modes breaks, or at least it persists only for a short time.

Let us now come to the dependence on the length $N$ of the chain, which represents the relevant information if one aims at investigating the thermodynamic limit. In fig. 3 we plot the calculated value of the size $v_{r}$ of the resonant packet as a function of the specific energy $\varepsilon$, in $\log -\log$ scale. For specific energies less than a threshold $\varepsilon^{*}$ (roughly between $10^{-3}$ and $10^{-2}$ ), the data points lie close to an unique line, thus indicating that the number of resonant modes belonging to the packet is proportional to $N$. Above the threshold the points start to fall below the interpolating line, and then appear to be scattered in some random way. For $\varepsilon$ slightly above $\varepsilon^{*}$ such behaviour is explained by the great sensitivity of our indicator with respect to the tail's growth in the energy spectrum; for higher specific energies instead the numerics confirms that the resonant packet is broken, and actually no meaningful information can be extracted from those data.

Recalling that the frequency spectrum of our model is given by $\omega=\sin (v \pi / 2)$ (up to a multiplicative constant) we conclude that our calculations confirm that we may identify a resonant packet that includes all modes up to a frequency $\omega_{r}(\varepsilon)$, as claimed in [5]. However a difference in the slope is observed, namely here we get $\omega_{r} \sim \varepsilon^{1 / 5}$ (roughly), to be compared with $\omega_{r} \sim \varepsilon^{1 / 4}$ observed in that paper. We have no definite explanation of this difference. However, we may add some remarks. First, the existence of packets of modes has been observed in other situations, with different initial conditions, and with different characteristics (e.g., in [26] it is even possible to find packets of modes for an $\alpha, \beta$ model with initial data in the high part of the spectrum). Secondly, different parameters have been

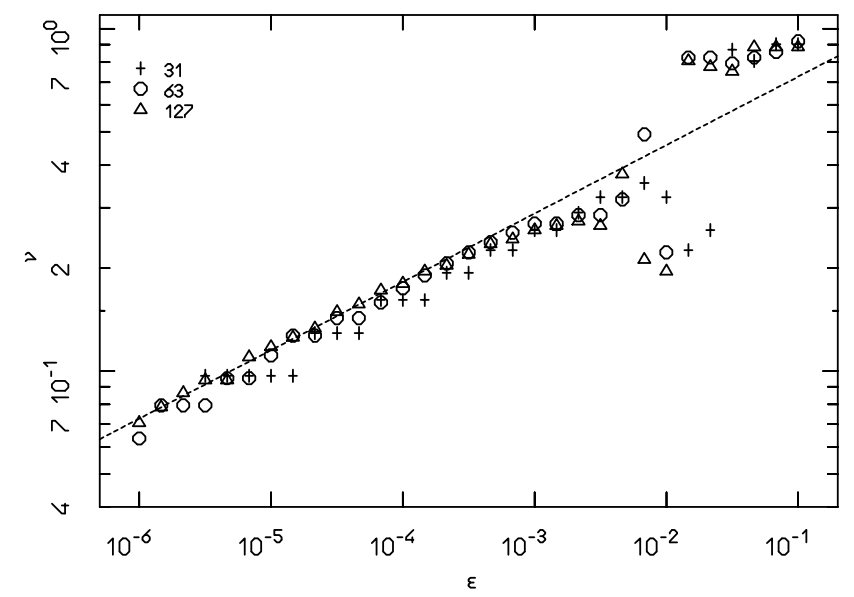

Figure 3: Plot of $v_{r}$ vs. $\varepsilon$, in $\log -\log$ scale. The symbols refer to $N=31$ (crosses), $N=63$ (circles) and $N=127$ (triangles); $T=4 . \times 10^{5}$. The dashed line has slope $1 / 5$.

used in order to evaluate the size of some packet of modes that interact in some sense, and moreover there is some arbitrary element in the choice of the parameters (e.g., in [5] and [26] one selects the modes that possess a fixed percent of the total energy, while in [2] the spectral entropy is used). Here, with respect to previous papers, we use a further different parameter which may be considered as defined in a more precise way, but is actually more difficult to calculate. Lastly, it could be necessary to explore higher values of $N$ to see a stabilized output of our numerical indicator. Thus, the difference in the observed slope may be due to several reasons that are hardly identified.

Concerning the second type of initial data considered, the outcome is qualitatively the same (pictures not shown), the only difference being in the exponent of the relation between the specific energy and the frequency threshold of the resonant packet: in this case we get $\omega_{r} \sim \varepsilon^{1 / 7}$. Although the second class of initial data could be reasonable for the above mentioned reasons, one also realizes that at low specific energies the expected natural packet of [5] is actually narrower than the group of modes initially excited in this case. In $[9,10]$ it is investigated the dynamics for this kind of initial conditions, and it turns out that actually there is no further spread of energy, with a decay of the energy spectrum depending on specific energy as a logarithm; thus one could also expect a much weaker dependence on $\varepsilon$ for the width of the metastable packet, compatible with the smaller exponent we find.

A last remark concerns the dependence of the threshold (in specific energy) on $N$. We could not identify a clear behaviour in the range of $N$ considered here. The data seem to indicate that the threshold slowly decreases with $N$. However, we should take into account that in previous calculation the same phenomenon has been observed, but the data appeared to become stable for higher values of $N$, e.g., for $N>1000$. Such a value can not be attained with our method.

\subsection{Toda's integrals}

Although it is not the scope of the present paper, it is rather 

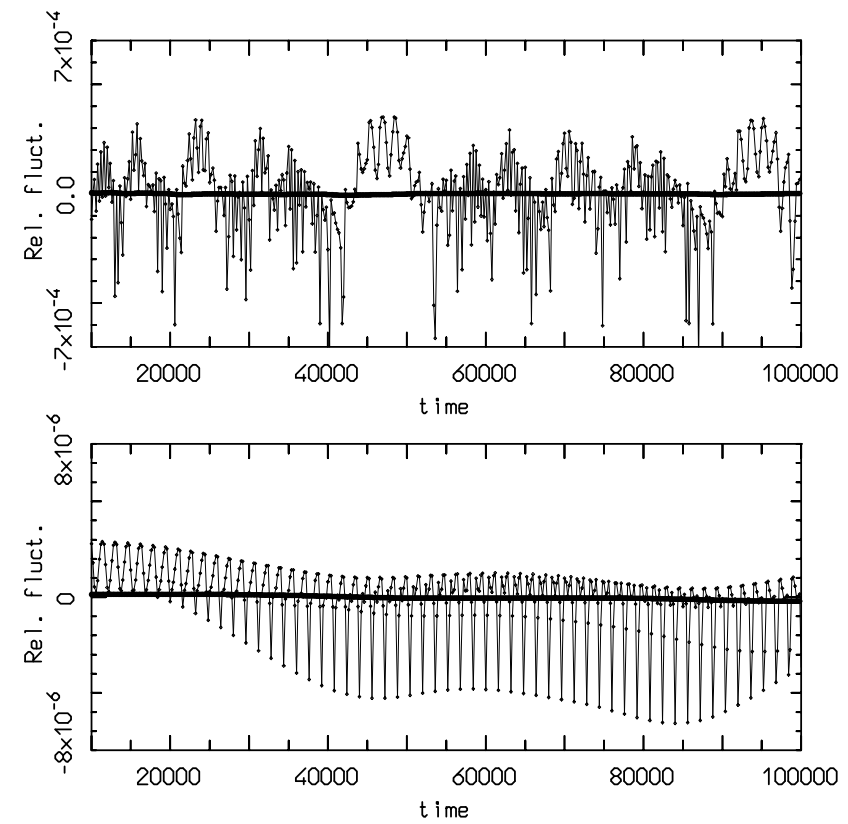

Figure 4: Plot of $\Phi(t) /\langle\Phi\rangle-1$ (thick line) and $J(t) /\langle J\rangle-1$ (thin line), for $t$ from $10^{4}$ to $10^{5} ; \beta=1 / 4$. Top panel: $\varepsilon=10^{-3}$. Bottom panel: $\varepsilon=10^{-5}$.

natural and surely interesting to ask whether there exists a relation between our approximate first integral $\Phi$ and (some of) the conserved quantities of the Toda model, which is the closest integrable system known.

Since it is clearly not relevant to match the actual constant value of the quantities at hand, we first tried to compare analitically at least their first order terms in a normal modes variable development, and then to investigate numerically their oscillations. As illustrated in [28], for fixed end conditions the first non trivial conserved quantity of the Toda model is $J_{4}$ (the notation is the same as in [22]); energy is denoted by $J_{2}$. Our claim is that precisely $J_{2}+J_{4}$ could have some interesting relation with $\Phi$. We will denote $J:=J_{2}+J_{4}$.

To support our claim we proceed as follows. Since one of the key point of our scheme is the resonance among the frequencies emerging from the quadratic term, we investigate those quantities. At the lowest order $\Phi$ has by construction the form $\sum_{j=1}^{n_{r}} j I_{j}$, with resonant frequencies $j$. The quadratic part of Toda's energy (wich coincide with the quadratic part of the FPU energy) has the form $\sum_{j=1}^{N} \omega_{j} I_{j}$, with frequencies, given by (5), linear in $j$ with a small correction starting with a term cubic in $j / N$. It happens that also $J_{4}$, in its quadratic part, has the same structure; for $J$ an exact compensation appears for those cubic terms, so that at first order it has the form $\sum_{j=1}^{N} v_{j} I_{j}$, with "frequencies" $v_{j}$ linear in $j$ plus a correction of the fifth order in $j / N$. Thus $J$, at least at first order, is definitely close to $\Phi$ with respect to its key resonance property.

It is then interesting to compare the relative fluctuations of both $\Phi$ and $J$ along the FPU dynamics. We have performed some simulations at different energy densities with both $\beta=$ $1 / 4$ and $\beta=\beta_{\text {Toda }}=2 / 3 \alpha^{2}$. The results obtained are shown, respectively, in Fig. 4 and Fig. 5, where the relative fluctuations
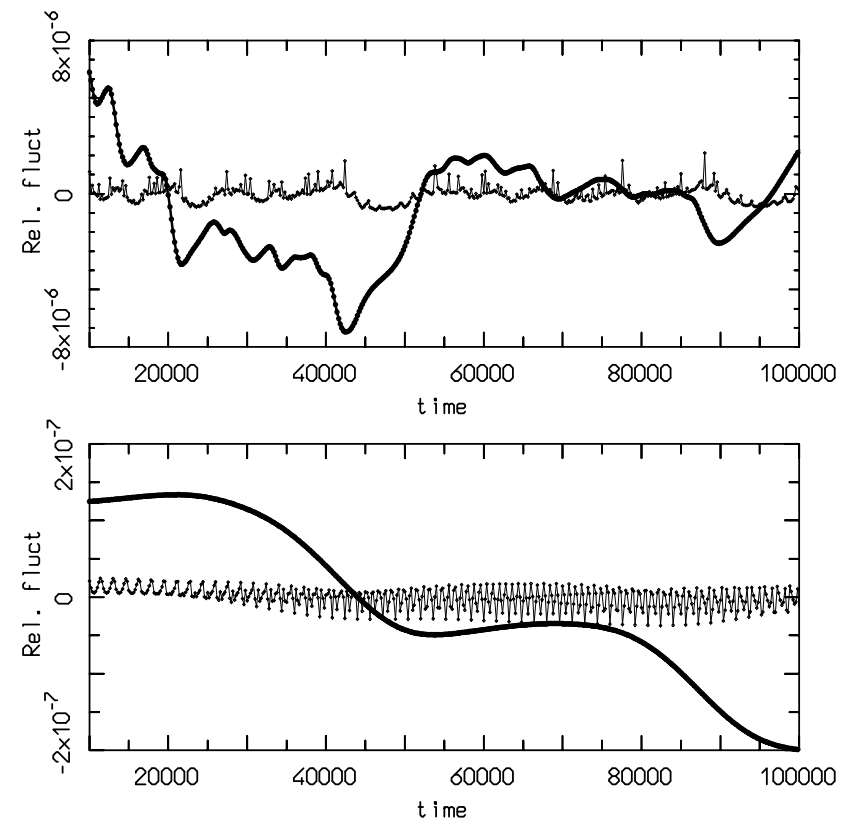

Figure 5: Plot of $\Phi(t) /\langle\Phi\rangle-1$ (thick line) and $J(t) /\langle J\rangle-1$ (thin line), for $t$ from $10^{4}$ to $10^{5} ; \beta=\beta_{\text {Toda }}$. Top panel: $\varepsilon=10^{-3}$. Bottom panel: $\varepsilon=10^{-5}$.

of $\Phi$ and $J$ (with respect to their time average) are compared on a time interval $\left[10^{4}, 10^{5}\right]$. Within the range of our numerical simulations, the fluctuation of the resonant adiabatic invariant $\Phi$ appears to be not affected by a moderate change in the quartic coefficient $\beta$, but it is very sensitive to the tuning of the energy density $\varepsilon$; indeed it comes from a perturbative construction (resonant normal form) which works on the cubic potential. On the other hand, the Toda integral $J$ looks less dependend on $\varepsilon$, but much more sensitive to small changes of $\beta$, and its fluctuation significantly decreases (especially at $\varepsilon=10^{-3}$ ) when $\beta=\beta_{\text {Toda }}$, which minimize the distance between FPU and Toda models. As a result, for the latter choice of $\beta$ the relative fluctuations of $\Phi$ and $J$ are comparable, while for $\beta=1 / 4$ the relative fluctuation of $\Phi$ is two order of magnitude smaller than that of $J$ for both the values of specific energy considered.

We may conclude that, except for values of $\beta$ equal or close to $\beta_{\text {Toda }}$, the resonant construction which generates $\Phi$ seems more suitable to the nonintegrable nature of the FPU system. However, in our opinion, the relation between the resonant adiabatic invariant we constructed, and the first integrals of the Toda lattice, deserves further investigation.

\section{Acknowledgment}

We thank G.Benettin, H.Christodoulidi and A.Ponno for the useful discussions on the role of initial data and on the conserved quantities of the Toda model; we also thank the referee for her/his suggestion to investigate the realtion between our approximate conserved quantity and Toda's integrals. SP and TP are partially supported by Indam-GNFM grant (Progetto giovani ricercatori) "Metodi perturbativi e multiscala in reticoli nonlineari". 


\section{References}

[1] Bambusi, D., Ponno, A.. On metastability in FPU. Comm Math Phys 2006;264(2):539-561.

[2] Benettin, G., Carati, A., Galgani, L., Giorgilli, A.. The Fermi-PastaUlam problem and the metastability perspective. In: The Fermi-PastaUlam problem. Berlin: Springer; volume 728 of Lecture Notes in Phys.; 2008. p. 152-189.

[3] Benettin, G., Livi, R., Ponno, A.. The Fermi-Pasta-Ulam problem: scaling laws vs. initial conditions. J Stat Phys 2009;135(5-6):873-893.

[4] Benettin, G., Ponno, A.. Time-scales to equipartition in the Fermi-PastaUlam problem: Finite-size effects and thermodynamic limit. Journal of Statistical Physics 2011;144:793-812. 10.1007/s10955-011-0277-9.

[5] Berchialla, L., Galgani, L., Giorgilli, A.. Localization of energy in FPU chains. Discrete Contin Dyn Syst 2004;11(4):855-866.

[6] Berchialla, L., Giorgilli, A., Paleari, S.. Exponentially long times to equipartition in the thermodynamic limit. Phys Lett A 2004;321(34):167-172.

[7] Birkhoff, G.D.. Dynamical systems. New York, N.Y., 1927.

[8] Bocchieri, P., Scotti, A., Bearzi, B., Loinger, A.. Anharmonic chain with Lennard-Jones interaction. Phys Rev A 1970;2:2013-2019.

[9] Christodoulidi, H., Efthymiopoulos, C., Bountis, T.. Energy localization on $q$-tori, long-term stability, and the interpretation of Fermi-Pasta-Ulam recurrences. Phys Rev E 2010;81:016210.

[10] Christodoulidi, H., Efthymiopoulos, C., Bountis, T. 2011;In preparation.

[11] Fermi, E., Pasta, J., Ulam, S.. Studies of nonlinear problems. In: Collected papers (Notes and memories). Vol. II: United States, 19391954. 1955. Los Alamos document LA-1940.

[12] Flach, S., Ivanchenko, M.V., Kanakov, O.I.. $q$-breathers in FermiPasta-Ulam chains: existence, localization, and stability. Phys Rev E (3) 2006;73(3):036618, 14.

[13] Gallavotti, G., editor. The Fermi-Pasta-Ulam problem. volume 728 of Lecture Notes in Physics. Berlin: Springer, 2008. A status report.

[14] Giorgilli, A.. A computer program for integrals of motion. Comp Phys Comm 1979;16:331-343.

[15] Giorgilli, A.. Small denominators and exponential stability: from Poincaré to the present time. Rend Sem Mat Fis Milano 1998;68:19-57 (2001).

[16] Giorgilli, A., Delshams, A., Fontich, E., Galgani, L., Simó, C.. Effective stability for a Hamiltonian system near an elliptic equilibrium point, with an application to the restricted three-body problem. J Differential Equations 1989;77(1):167-198.

[17] Giorgilli, A., Galgani, L.. Formal integrals for an autonomous Hamiltonian system near an equilibrium point. Celestial Mech 1978;17(3):267280.

[18] Giorgilli, A., Muraro, D.. Exponentially stable manifolds in the neighbourhood of elliptic equilibria. Boll Unione Mat Ital Sez B Artic Ric Mat (8) 2006;9(1):1-20.

[19] Giorgilli, A., Paleari, S., Penati, T.. Local chaotic behaviour in the FPU system. Discrete Contin Dyn Syst Ser B 2005;5(4):991-1004.

[20] Gustavson, F.G.. On constructing formal integrals of a Hamiltonian system near an equilibrium point. Astron J 1966;71:670-686.

[21] Hairer, E., Lubich, C.. On the energy distribution in Fermi-Pasta-Ulam lattices 2011; Preprint.

[22] Hénon, M.. Integrals of the Toda lattice. Phys Rev B 1974;9(4):19211923.

[23] Izrailev, F.M., Chirikov, B.V.. Statistical properties of a nonlinear string. Sov Phys Dokl 1966;11(1):30-32.

[24] Lichtenberg, A.J., Livi, R., Pettini, M., Ruffo, S.. Dynamics of oscillator chains. In: The Fermi-Pasta-Ulam problem. Berlin: Springer; volume 728 of Lecture Notes in Phys.; 2008. p. 21-121.

[25] Lorenzoni, P., Paleari, S.. Metastability and dispersive shock waves in the Fermi-Pasta-Ulam system. Phys D 2006;221(2):110-117.

[26] Paleari, S., Penati, T.. Equipartition times in a Fermi-Pasta-Ulam system. Discrete Contin Dyn Syst 2005;(suppl.):710-720. Dynamical systems and differential equations (Pomona, CA, 2004).

[27] Penati, T., Flach, S.. Tail resonances of Fermi-Pasta-Ulam $q$ breathers and their impact on the pathway to equipartition. Chaos 2007; 17(2):023102, 16.

[28] Ponno, A., Christodoulidi, H., Skokos, C., Flach, S.. The twostage dynamics in the Fermi-Pasta-Ulam problem: From regular to diffu- sive behavior. Chaos: An Interdisciplinary Journal of Nonlinear Science 2011;21(4):043127. 